25 March 1998

\title{
A study of pseudoscalar states produced centrally in pp interactions at $450 \mathrm{GeV} / \mathrm{c}$
}

\author{
The WA102 Collaboration
}

\begin{abstract}
D. Barberis ${ }^{5}$, W. Beusch ${ }^{5}$, F.G. Binon ${ }^{7}$, A.M. Blick ${ }^{6}$, F.E. Close $^{4}$, K.M. Danielsen ${ }^{12}$, A.V. Dolgopolov ${ }^{6}$, S.V. Donskov ${ }^{6}$, B.C. Earl ${ }^{4}$, D. Evans $^{4}$, B.R. French ${ }^{5}$, T. Hino ${ }^{13}$, S. Inaba ${ }^{9}$, A.V. Inyakin ${ }^{6}$, T. Ishida ${ }^{9}$, A. Jacholkowski ${ }^{5}$, T. Jacobsen ${ }^{12}$, G.T Jones ${ }^{4}$, G.V. Khaustov ${ }^{6}$, T. Kinashi ${ }^{14}$, J.B. Kinson ${ }^{4}$, A. Kirk ${ }^{4}$, W. Klempt ${ }^{5}$, V. Kolosov ${ }^{6}$, A.A. Kondashov ${ }^{6}$, A.A. Lednev ${ }^{6}$, V. Lenti ${ }^{5}$, S. Maljukov ${ }^{8}$, P. Martinengo ${ }^{5}$, I. Minashvili ${ }^{8}$, K. Myklebost ${ }^{3}$, T. Nakagawa ${ }^{13}$, K.L. Norman ${ }^{4}$, J.M. Olsen ${ }^{3}$, J.P.Peigneux ${ }^{1}$, S.A. Polovnikov ${ }^{6}$, V.A. Polyakov ${ }^{6}$, V. Romanovsky ${ }^{8}$, H. Rotscheidt ${ }^{5}$, V. Rumyantsev ${ }^{8}$, N. Russakovich ${ }^{8}$, V.D. Samoylenko ${ }^{6}$, A. Semenov ${ }^{8}$, M. Sené ${ }^{5}$, R. Sené ${ }^{5}$, P.M. Shagin ${ }^{6}$, H. Shimizu ${ }^{14}$, A.V.Singovsky ${ }^{1,6}$, A. Sobol ${ }^{6}$, A. Solovjev ${ }^{8}$, M. Stassinaki ${ }^{2}$, J.P. Stroot ${ }^{7}$, V.P. Sugonyaev ${ }^{6}$, K. Takamatsu ${ }^{10}$, G. Tchlatchidze ${ }^{8}$, T. Tsuru ${ }^{9}$, M. Venables ${ }^{4}$, O. Villalobos Baillie ${ }^{4}$, M.F. Votruba ${ }^{4}$, Y. Yasu ${ }^{9}$.
\end{abstract}

\begin{abstract}
A study has been made of pseudoscalar mesons produced centrally in pp interactions. The results show that the $\eta$ and $\eta^{\prime}$ appear to have a similar production mechanism which differs from that of the $\pi^{0}$. The production properties of the $\eta$ and $\eta^{\prime}$ are not consistent with what is expected from double Pomeron exchange. In addition the production mechanism for the $\eta$ and $\eta^{\prime}$ is such that the production cross section are greatest when the azimuthal angle between the $p_{T}$ vectors of the two protons is 90 degrees.
\end{abstract}

Submitted to Physics Letters

\footnotetext{
LAPP-IN2P3, Annecy, France.

Athens University, Physics Department, Athens, Greece.

Bergen University, Bergen, Norway.

School of Physics and Astronomy, University of Birmingham, Birmingham, U.K.

CERN - European Organization for Nuclear Research, Geneva, Switzerland.

IHEP, Protvino, Russia.

IISN, Belgium.

JINR, Dubna, Russia.

9 High Energy Accelerator Research Organization (KEK), Tsukuba, Ibaraki 305, Japan.
} 
Faculty of Engineering, Miyazaki University, Miyazaki, Japan.

RCNP, Osaka University, Osaka, Japan.

2 Oslo University, Oslo, Norway.

13 Faculty of Science, Tohoku University, Aoba-ku, Sendai 980, Japan.

14 Faculty of Science, Yamagata University, Yamagata 990, Japan. 
The WA102 experiment is designed to study the properties of centrally produced mesons. Reactions of this type are expected to be mediated by double exchange processes, with a mixture of Pomeron-Pomeron, Reggeon-Pomeron and Reggeon-Reggeon exchange. In order to try to understand the nature of the centrally produced mesons it is interesting to try to determine which exchange process is producing them. For instance, the Pomeron is thought to be a multigluonic object and consequently it has been anticipated that the production of glue rich states may be especially favoured in Double Pomeron Exchange (DPE). It has been proposed that the $\eta$ and $\eta^{\prime}$ are strongly connected to glue [1]. Further information on this subject could come from studying if the $\eta$ or $\eta^{\prime}$ are produced by DPE.

It has previously been reported that in central production $0^{-+}$states are suppressed relative to $1^{++}$states [2]. In addition, both the $\eta$ and $\eta^{\prime}$ signals are suppressed at small four-momentum transfers where DPE is believed to be dominant [2, 3]. Hence it could be conjectured that $0^{-+}$ objects do not couple to DPE. This hypothesis has been further tested by measuring the cross section of the production of the $\eta$ and $\eta^{\prime}$ as a function of energy [4, 5]. The result shows that the cross section for production of these resonances decreases with energy which is inconsistent with production via DPE which is predicted to give a constant cross section.

This paper presents new results from the WA102 experiment on the centrally produced $\pi^{0}$, $\eta$ and $\eta^{\prime}$ formed in the reactions

$$
\begin{gathered}
p p \rightarrow p_{f}\left(\pi^{0} \rightarrow \gamma \gamma\right) p_{s} \\
p p \rightarrow p_{f}(\eta \rightarrow \gamma \gamma) p_{s} \\
p p \rightarrow p_{f}\left(\eta \rightarrow \pi^{+} \pi^{-} \pi^{0}, \pi^{0} \rightarrow \gamma \gamma\right) p_{s} \\
p p \rightarrow p_{f}\left(\eta^{\prime} \rightarrow \pi^{+} \pi^{-} \eta, \eta \rightarrow \gamma \gamma\right) p_{s}
\end{gathered}
$$

at $450 \mathrm{GeV} / \mathrm{c}$. The subscripts $\mathrm{f}$ and $\mathrm{s}$ indicate the fastest and slowest particles in the laboratory respectively. The data come from experiment WA102 which has been performed using the CERN Omega Spectrometer. The layout of the Omega Spectrometer used in this run is similar to that described in ref. [6] with the replacement of the OLGA calorimeter by GAMS 4000 [7].

Reactions (11)-(4) have been isolated from the sample of events having the required number of outgoing charged tracks plus two $\gamma$ s reconstructed in the electromagnetic calorimeter by first imposing the following cuts on the components of the missing momentum: $\mid$ missing $P_{x} \mid<17.0$ $\mathrm{GeV} / \mathrm{c}, \mid$ missing $P_{y} \mid<0.16 \mathrm{GeV} / \mathrm{c}$ and $\mid$ missing $P_{z} \mid<0.12 \mathrm{GeV} / \mathrm{c}$, where the $\mathrm{x}$ axis is along the beam direction. A correlation between pulse-height and momentum obtained from a system of scintillation counters was used to ensure that the slow particle was a proton. Evidence of proton excitation can be observed in the $p_{f} \pi^{0}$ mass spectrum (i.e. $\Delta^{+}$or $N^{*+}$ production) which has been removed by requiring $M\left(p_{f} \pi^{0}\right)>2.0 \mathrm{GeV}$.

Fig. (1a) and b) shows the two photon mass spectra in the region of the $\pi^{0}$ and $\eta$ respectively for reactions 1 and 2. The mass resolution is $\sigma=14 \mathrm{MeV}$ for the $\pi^{0}$ and $\sigma=33 \mathrm{MeV}$ for the $\eta$. Fig. [1 c) shows the $\pi^{+} \pi^{-} \pi^{0}$ mass spectrum in the region of $\eta$ for reaction (3) where the $\pi^{0}$ is detected decaying to two photons and kinematically fitted to give the $\pi^{0}$ mass. The mass resolution is $\sigma=11 \mathrm{MeV}$. Fig. 11d) shows the $\eta \pi^{+} \pi^{-}$mass spectrum in the region of $\eta^{\prime}$ for

\footnotetext{
${ }^{1}$ The showers associated with the impact of the charged tracks on the calorimeter have been removed from the event before the requirement of only two $\gamma \mathrm{s}$ was made.
} 
reaction (四) where the $\eta$ is detected decaying to two photons and kinematically fitted to give the $\eta$ mass. The mass resolution is $\sigma=10 \mathrm{MeV}$.

A Monte Carlo simulation has been performed to calculate the acceptance for each decay mode. The acceptance corrected Feynman $\mathrm{x}\left(x_{F}\right)$ distributions are shown in fig. 2a), b) and c) for the $\pi^{0}, \eta$ and $\eta^{\prime}$ respectively for $x_{F} \geq 0.0$. A cut of $0.0 \leq x_{F} \leq 0.1$ has been used to select central events in a region where the acceptance is effectively flat for all three mesons.

After acceptance correction the branching ratio of the $\eta$ to $2 \gamma$ and $\pi^{+} \pi^{-} \pi^{0}$ has been calculated to be

$$
\frac{\eta \rightarrow \gamma \gamma}{\eta \rightarrow \pi^{+} \pi^{-} \pi^{0}}=1.60 \pm 0.05 \pm 0.08
$$

which is consistent with the PDG [8] value of $1.69 \pm 0.04$. The systematic error is due mainly to the different trigger efficiencies for the decay of central systems to all photon final states relative to final states involving charged particles. Since the background below the $\eta$ decay to $\pi^{+} \pi^{-} \pi^{0}$ is smaller than in the $2 \gamma$ decay mode, it is the data from the $\pi^{+} \pi^{-} \pi^{0}$ decay mode which will be used in the following analysis. However, the $2 \gamma$ decay mode gives consistent results.

It has recently been suggested [9, 10] that the central production of pseudoscalars could be explained if the production mechanism is through the fusion of two vectors. In particular ref. [10] has suggested that $\gamma \gamma$ fusion may be the dominant mechanism for pseudoscalars in central production. To test this hypothesis, and provide further information on their production properties, the cross sections for the production of pseudoscalar mesons have been calculated for the Feynman $\mathrm{x}$ interval $0.0 \leq x_{F} \leq 0.1$ taking into account the unseen decay modes. The resulting cross sections are

$$
\begin{array}{llll}
\sigma\left(\pi^{0}\right) & = & 17609 \pm 210 \pm 2400 & \mathrm{nb} \\
\sigma(\eta) & = & 1295 \pm 16 \pm 120 & \mathrm{nb} \\
\sigma\left(\eta^{\prime}\right) & = & 588 \pm 18 \pm 60 & \mathrm{nb} .
\end{array}
$$

The dominant contribution to the systematic error comes from the uncertainty in the sensitivity of the experiment. These cross sections are several orders of magnitude larger than what would be expected for $\gamma \gamma$ fusion which we have calculated, using the method described in ref. [11], to be less than $1 \mathrm{nb}$ in the kinematic window considered.

The relative cross sections can be measured more precisely due to the cancellation of common systematic errors and are

$$
\sigma\left(\pi^{0}\right): \sigma(\eta): \sigma\left(\eta^{\prime}\right)=1: 0.073 \pm 0.005: 0.033 \pm 0.002
$$

These ratios are in agreement with $\sigma\left(\pi^{0}\right): \sigma(\eta): \sigma\left(\eta^{\prime}\right)=1: 0.080 \pm 0.015: 0.028 \pm 0.009$ found previously by the NA12/2 experiment studying the reaction $\pi^{-} N \rightarrow \pi^{-} N X^{0}$ at $300 \mathrm{GeV} / \mathrm{c}$ incident beam momentum [12]. It is interesting to note that the NA12/2 experiment found that the $x_{F}$ dependence of the $\pi^{0}, \eta$ and $\eta^{\prime}$ was effectively flat in the range $0.0 \leq x_{F} \leq 0.3$ in contrast to what is observed in this current experiment (see fig. 20 a), b) and c)).

In previous analyses of other channels it has been observed that when the centrally produced system has been analysed as a function of the parameter $d P_{T}$, which is the difference in the transverse momentum vectors of the two exchange particles [13], all the undisputed $q \bar{q}$ states 
are suppressed at small $d P_{T}$. Therefore, a study of the centrally produced pseudoscalar states has been performed as a function of $d P_{T}$. Fig. 2 $2 \mathrm{~d}$ ), e) and f) shows the $d P_{T}$ spectra for the $\pi^{0}$, $\eta$ and $\eta^{\prime}$ respectively. The $d P_{T}$ spectra for the $\eta$ and $\eta^{\prime}$ are very similar; however, the $\pi^{0}$ has a different spectrum with more events at small $d P_{T}$. Table 1 gives the fraction of each resonance in three bins of $d P_{T}$. As can be seen all the states are suppressed at small $d P_{T}$ consistent with what has been observed previously for all other $q \bar{q}$ states [13]. This suppression of pseudoscalar states at small $d P_{T}$ is consistent with their production through vector vector fusion [9, 10].

Fig. (3a), b) and c) shows the four momentum transfered from one of the proton vertices for the $\pi^{0}, \eta$ and $\eta^{\prime}$ respectively. None of these distributions can be fitted with a single exponential. The $\eta$ and $\eta^{\prime}$ have a similar distribution while the $\pi^{0}$ is different. It should be noted that the $t$ spectra for the $\eta$ and $\eta^{\prime}$ are not consistent with what is expected from DPE, namely an $e^{-b t}$ behaviour with $b \approx 6-8 \mathrm{GeV}^{-2}$ [14]. The t distributions have been fitted to the form

$$
\frac{d \sigma}{d t}=\alpha e^{-b_{1} t}+\beta t^{n} e^{-b_{2} t}
$$

where values of $n$ of 1 or 2 have been tried. Fits with $n=2$ were found to give the best fit to the data. The parameters resulting from the fit are given in table 2. As can be seen the values of $b_{1}$ and $b_{2}$ are consistent in each case. The main difference is that the $\pi^{0}$ has a larger value of the ratio $\alpha / \beta$.

The azimuthal angle $(\phi)$ between the $p_{T}$ vectors of the two protons, which is shown in fig. (3d), e) and f), shows a very interesting effect. The $\eta$ and $\eta^{\prime}$ are produced predominantly when the angle $\phi$ is 90 degrees.

In summary, it would appear that the $\eta$ and $\eta^{\prime}$ have a similar production mechanism which differs from that of the $\pi^{0}$. The $t$ spectra for the $\eta$ and $\eta^{\prime}$ are not consistent with what is expected from DPE and this fact together with the previous observation that the energy dependence of

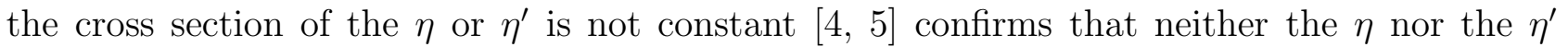
are produced dominantly by DPE. In addition the production mechanism for the $\eta$ and $\eta^{\prime}$ is such that the production cross sections are greatest when the azimuthal angle between the $p_{T}$ vectors of the two protons is 90 degrees.

\section{Acknowledgements}

This work is supported, in part, by grants from the British Particle Physics and Astronomy Research Council, the British Royal Society, the Ministy of Education, Science, Sports and Culture of Japan (grants no. 04044159 and 07044098) and the Russian Foundation for Basic Research (grant 96-15-96633). 


\section{References}

[1] V.A. Novikov et al.,, Nucl. Phys. B191 (1981) 301;

J. Rosner, Phys. Rev. D27 (1983) 1101;

S.S. Gershtein, A.A. Likhoded and Yu. D. Prokoshkin, Zeit. Phys. C24 (1984) 305;

R. Akhoury and J.-M. Frere, Phys. Lett B220 (1989) 258;

P. Ball, J.-M. Frere and M. Tytgat, Phys. Lett B365 (1996) 367.

[2] T. Armstrong et al., Zeit. Phys. C52 (1991) 389.

[3] T. Armstrong et al., Zeit. Phys. C48 (1990) 213.

[4] D. Barberis et al., hep-ex/9801003, To be published in Phys. Lett.

[5] F.E. Close and A. Kirk, Zeit. Phys. C76 (1997) 469.

[6] F. Antinori et al., Il Nuovo Cimento A107 (1994) 1857.

[7] D. Alde et al., Nucl. Phys. B269 (1986) 485.

[8] Particle Data Group, Phys Rev. D54 (1996) 1.

[9] F.E. Close, hep-ph/9710450.

[10] P. Castoldi, R. Escribano and J.-M. Frere, hep-ph/9712387.

[11] W. Kilian and O. Nachtmann, hep-ph/9712371.

[12] D. Alde et al., Zeit. Phys. C43 (1989) 541.

[13] D. Barberis et al., Phys. Lett. B397 (1997) 339.

[14] S.N. Ganguli and D.P. Roy, Phys. Rep. 67 (1980) 203. 


\section{Tables}

Table 1: Resonance production as a function of $d P_{T}$ expressed as a percentage of its total contribution.

\begin{tabular}{|c|c|c|c|}
\hline & $d P_{T} \leq 0.2 \mathrm{GeV}$ & $0.2 \leq d P_{T} \leq 0.5 \mathrm{GeV}$ & $d P_{T} \geq 0.5 \mathrm{GeV}$ \\
\hline$\pi^{0}$ & $13 \pm 1 \pm 2$ & $45 \pm 1 \pm 2$ & $42 \pm 1 \pm 4$ \\
\hline$\eta$ & $6 \pm 1 \pm 2$ & $34 \pm 1 \pm 2$ & $60 \pm 1 \pm 3$ \\
\hline$\eta^{\prime}$ & $3 \pm 1 \pm 2$ & $33 \pm 1 \pm 2$ & $64 \pm 1 \pm 3$ \\
\hline
\end{tabular}


Table 2: Parameters from the fit to the $\mathrm{t}$ distribution

\begin{tabular}{|c|c|c|c|c|}
\hline & $\alpha \times 10^{3}$ & $b_{1} \mathrm{GeV}^{-2}$ & $\beta \times 10^{3}$ & $b_{2} \mathrm{GeV}^{-2}$ \\
\hline$\pi^{0}$ & $128 \pm 9$ & $14.9 \pm 0.3$ & $411 \pm 61$ & $12.3 \pm 0.4$ \\
\hline$\eta$ & $2.3 \pm 0.3$ & $15 \pm 5$ & $215 \pm 12$ & $11.6 \pm 0.2$ \\
\hline$\eta^{\prime}$ & $0.3 \pm 0.2$ & $15 \pm 5$ & $108 \pm 8$ & $11.2 \pm 0.2$ \\
\hline
\end{tabular}




\section{Figures}

Figure 1: The effective mass spectra for the reaction $p p \rightarrow p_{f} p_{s} X^{0}$ with $X^{0}$ decaying to a), b) $\gamma \gamma$, c) $\pi^{+} \pi^{-} \pi^{0}$ and d) $\eta \pi^{+} \pi^{-}$.

Figure 2: a), b) and c) The $x_{F}$ distribution for the $\pi^{0}, \eta$ and $\eta^{\prime}$ respectively. d), e) and f) The $d P_{T}$ spectra for the $\pi^{0}, \eta$ and $\eta^{\prime}$ respectively.

Figure 3: The four momentum transfer squared $(|t|)$ from one of the proton vertices for the a) $\pi^{0}$, b) $\eta$ and c) and $\eta^{\prime}$. The azimuthal angle $(\phi)$ between the two outgoing protons for the d) $\pi^{0}$, e) $\eta$ and f) and $\eta^{\prime}$. 

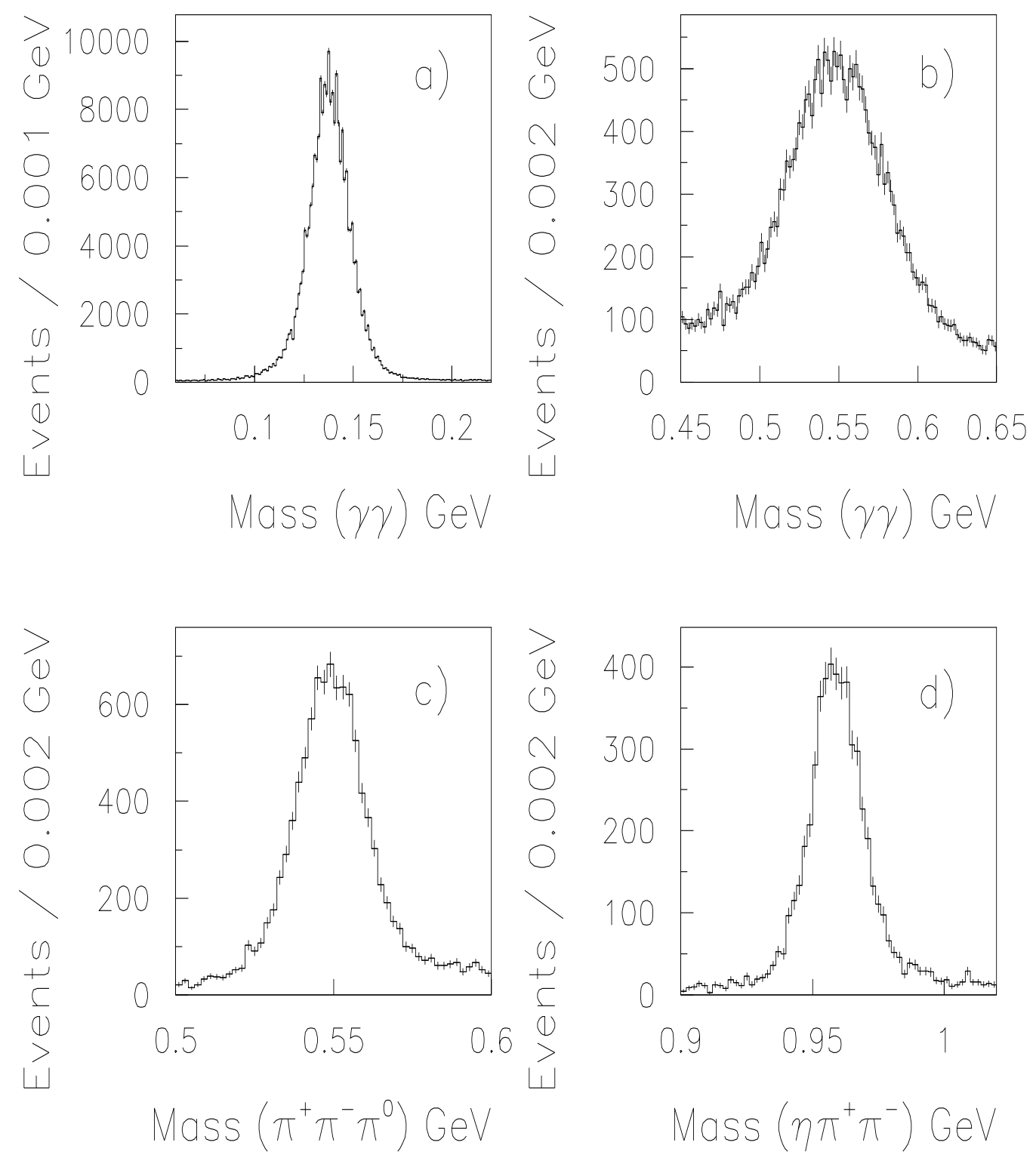

Figure 1 

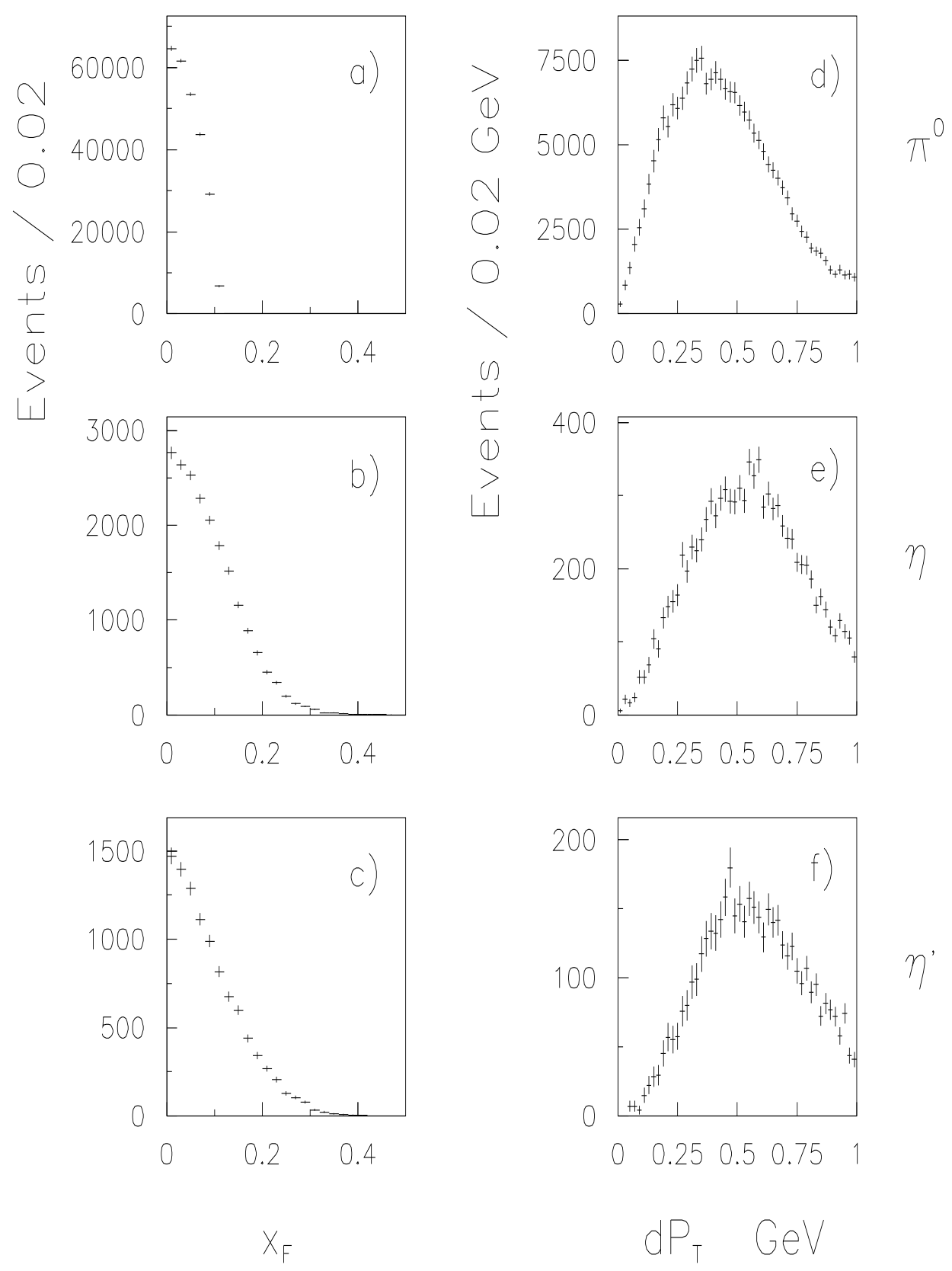

Figure 2 

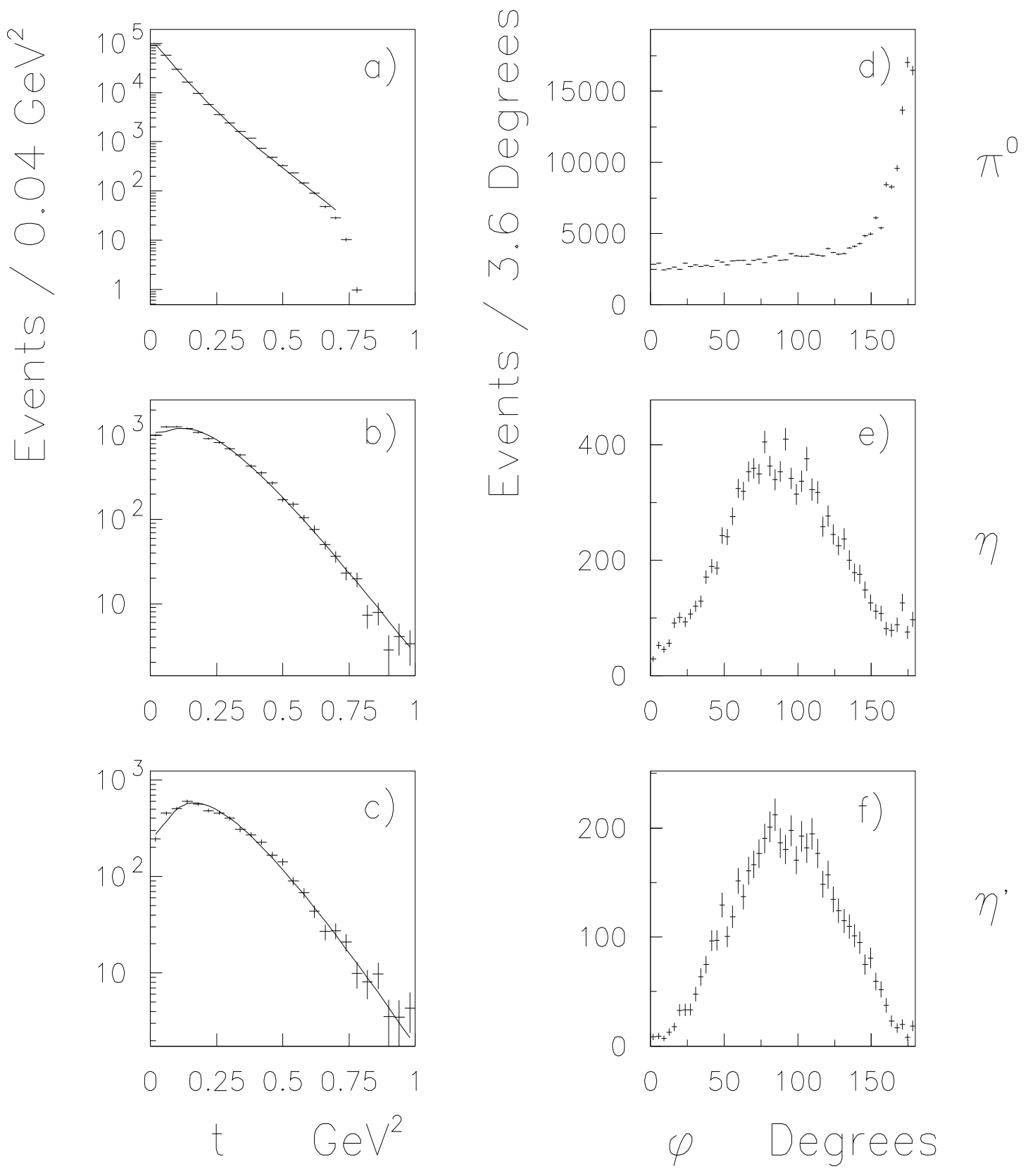

Figure 3 